\title{
Norovirus infection - frequency and duration of viral shedding in paediatric haemato-/oncologic patients
}

\section{Medical University of Graz}

Markus Keldorfer ${ }^{1}$, Klara Zach ${ }^{1}$, Harald H. Kessler ${ }^{2}$, Evelyn Stelz $\left.\right|^{2}$, Anna Karastaneva1 ${ }^{1}$, Daniela Sperl ${ }^{1}$, Herwig Lackner ${ }^{1}$, Martin Benesch ${ }^{1}$, Volker Strenger $^{1}$ ${ }^{1}$ Department of Paediatrics and Adolescent Medicine, Medical University of Graz, Graz, Austria

2 Institute of Hygiene, Microbiology and Environmental Medicine, Medical University of Graz, Graz, Austria

\section{Background}

Noroviruses (NV) cause severe gastrointestinal infections. Incidence and duration of shedding in paediatric haemato-/oncologic patients has not been studied so far.

\section{Materials/methods}

We retrospectively analysed results of NV reverse transcription PCRs from stool specimens obtained from haemato-/oncologic patients from 2010 to 2016. NV PCRs were performed as weekly screening in stem cell transplantation recipients or in patients with symptoms of gastroenteritis as well as in patients with prior NV detection.

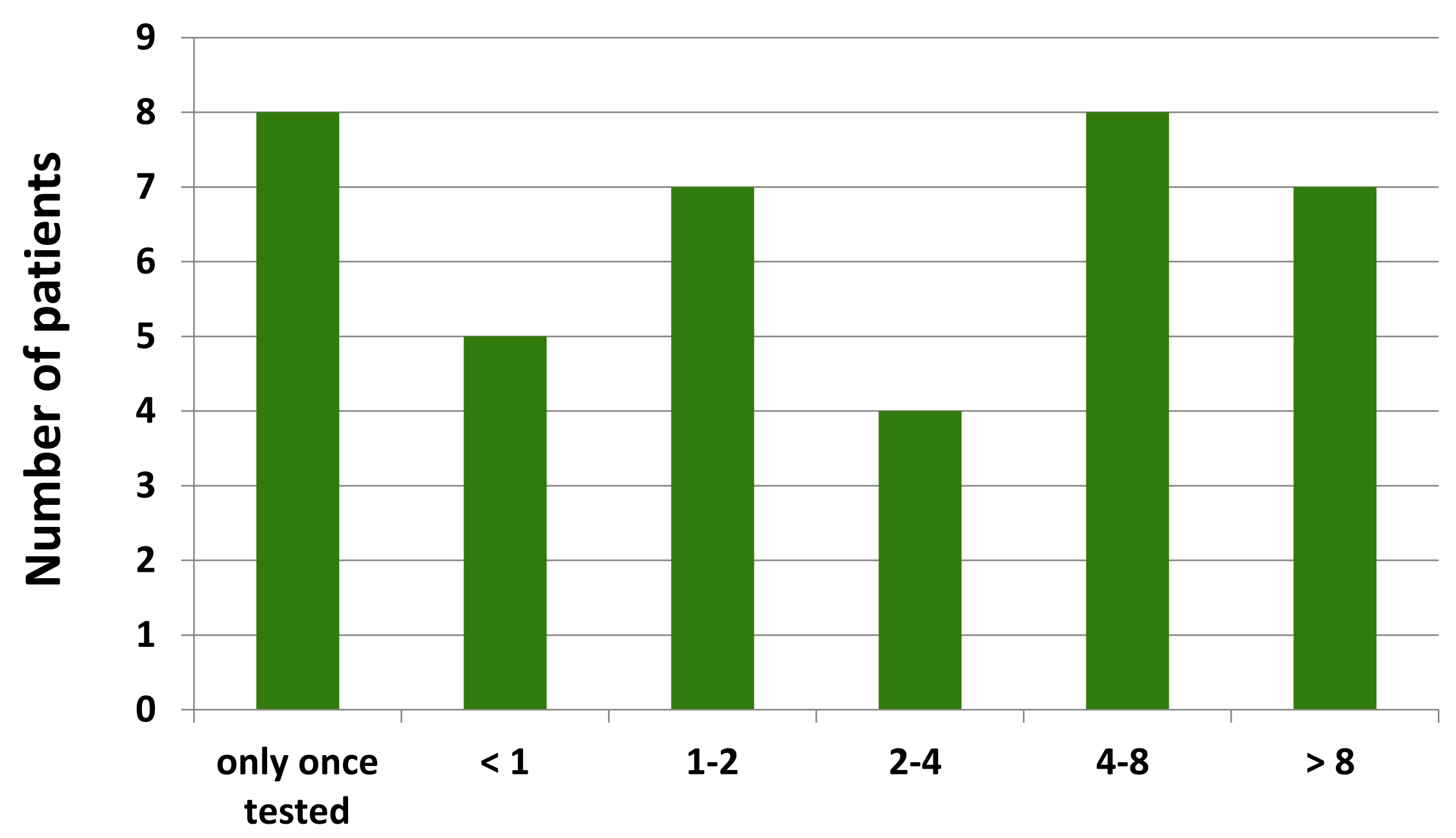

Documented time of viral shedding in weeks

\section{Results}

997 results from 152 patients (age 0.2-33.6, median 8.2 years, female 44.7\%) were evaluable of which 241 PCRs (24.2\%) were positive for NV.

$\mathbf{3 9} / \mathbf{1 5 2}$ patients $(\mathbf{2 5 . 7 \%}$, age $0.3-21.5$, median 6.9 years, $33.3 \%$ female) were tested positive at least once.

In 23 (59.0\%) of 39 positive tested patients, end of shedding could be defined and was documented for $\mathbf{2}$ to $\mathbf{1 7 0}$ (median 25) days.

In 7/39 (17.9\%) positive tested patients end of shedding could not be defined (despite multiple testing), with documented shedding for at least 4 to 160 (median 11) days. A 15-year-old female patient with primary immunodeficiency has been shedding up to now for at least 5.3 years.

In 8/39 (20.5\%) positive tested patients, no follow-up examination was performed.

In $19 / 39$ positive tested patients (48.7\%), shedding of more than 2 weeks and in $15 / 39$ patients $(38.5 \%)$ shedding of more than 4 weeks was documented without persistence of norovirus-typical symptoms during this period.

\section{Conclusion}

NVs in stool were detected in a quarter of patients tested. Duration of shedding was variable and was documented for up to 6 months for one patient longer than 5 years. Nearly $40 \%$ of the positive tested patients showed shedding of more than 4 weeks. These mostly asymptomatic shedders may be a major source of infection for other patients. Their significance in clinical management and infection control must be investigated in further studies. 